\title{
Fibroscan and FIB-4 Assessment Versus Lymphocyte I Monocyte Ratio as Predictors of Post-Hepatectomy Liver Failure in HCV Egyptian Patients with Hepatocellular Carcinoma
}

\author{
Mona Nasef ${ }^{1, ~ *, ~ L o b n a ~ A b o ~ A l i ~}{ }^{1}$, Mohammed Hablus ${ }^{2}$, Hossam Eldeen Soliman ${ }^{3}$, Nehad Hawash ${ }^{1}$, \\ Nadia Elwan ${ }^{1}$ \\ ${ }^{1}$ Tropical Medicine and Infectious Diseases Department, Faculty of Medicine, Tanta University, Tanta, Egypt \\ ${ }^{2}$ Gastrointestinal, Liver and Laparoscopic Surgery Department, Faculty of Medicine, Tanta University, Tanta, Egypt \\ ${ }^{3} \mathrm{HPB}$ and Liver transplantation surgery Department, National Liver Institute, Menoufia University, Menoufia, Egypt
}

\section{Email address:}

mona.nasef@med.tanta.edu.eg (M. Nasef), Mohamed.habls@med.tanta.edu.eg (M. Hablus), lobna.aboali@med.tanta.edu.eg (H. E. Soliman), Hosam123mail@yahoo.com (H. E. Soliman), nehad.hawash@med.tanta.edu.eg (N. Hawash), nad.dec1983@gmail.com (N. Elwan) *Corresponding author

\section{To cite this article:}

Mona Nasef, Lobna Abo Ali, Mohammed Hablus, Hossam Eldeen Soliman, Nehad Hawash, Nadia Elwan. Fibroscan and FIB-4 Assessment Versus Lymphocyte / Monocyte Ratio as Predictors of Post-Hepatectomy Liver Failure in HCV Egyptian Patients with Hepatocellular Carcinoma. International Journal of Medical Imaging. Vol. 8, No. 4, 2020, pp. 93-101. doi: 10.11648/j.ijmi.20200804.15

Received: October 20, 2020; Accepted: November 10, 2020; Published: November 24, 2020

\begin{abstract}
Background: Post-hepatectomy liver failure (PHLF) is the most leading cause of mortality in patients diagnosed with hepatocellular carcinoma (HCC) and undergoing resection of the affected part of the liver. Objectives: This research aimed to determine the value of the lymphocyte to monocyte ratio (LMR), fibrosis score 4 (FIB-4) and liver stiffness measurements (LSM) using Fibroscan as pre-operative predictors of PHLF in Egyptian patients with post- hepatitis C virus (HCV) liver cirrhosis and HCC. Methods: In this prospective cohort study definition of PHLF was done according to the "5050 criteria". Multivariate analysis was done to identify PHLF independent predictors. The predictive accuracy of the preoperative LMR, FIB-4 and LSM with Fibroscan were evaluated by receiver operating characteristic (ROC) curve. Results: Enrollment of fifty Egyptian patients was done. 14 patients (28\%) experienced PHLF. The presence of oesophageal varices, serum AST, serum albumin, LMR, FIB-4 score, and LSM $(\mathrm{P}<0.05)$ were independent pre-operative predictors for PHLF. According to ROC curve analysis, LMR yielded the best accuracy for predicting PHLF at cutoff $<3.33$ [AUC $=0.940$; sensitivity $=93.65 \%$; specificity $=94.44 \%$; positive predictive value $=86.67 \%$; negative predictive value $=97.14 \%]$. FIB-4 score and LSM had lower AUC (0.886 and 0.875) respectively. Conclusion: The pre-operative LMR has a higher predictive ability for PHLF in patients with HCV-related HCC undergoing hepatectomy compared with FIB-4 score and LSM using Fibroscan.
\end{abstract}

Keywords: Hepatocellular Carcinoma, Resection, Post-hepatectomy Liver Failure, Lymphocyte to Monocyte Ratio, Fibroscan, Fibrosis Score 4

\section{Introduction}

An epidemiological study from Egypt had revealed that liver cancer forms; $1.68 \%$ of the total malignancies, $11.75 \%$ of the malignancies of all digestive organs and $70.48 \%$ of all liver tumors. Hepatocellular carcinoma (HCC) is considered as a primary cancer of the liver arising from hepatocytes and accounting for 85 to $90 \%$ of the entire primary liver cancers [1].

One of the potential curative therapies for $\mathrm{HCC}$ is liver resection. But its feasibility is limited in the majority of those patients as most of them have associated chronic liver diseases increasing the risk of post-operative morbidity and mortality [2]. 
Post-hepatectomy liver failure (PHLF) had been identified as a severe post-operative complication and is considered as the main cause of death following resection of the liver. That is strongly related to cirrhosis, limited residual liver tissue and relatively poor liver regeneration ability. Even with major advances in technology and increasing experience in centers specialized for liver resection; PHLF is still a major problem due to associated high rates of post-operative morbidity $(4.09 \%-47.7 \%)$ and mortality $(0.24 \%-9.7 \%)$ [3].

Various laboratory, imaging techniques and indices have been proposed for proper pre-operative assessment of hepatic functional reserve including serum hyaluronic acid assay, volumetric assessment of the remnant liver, indocyanine green clearance test which is the most commonly used method for quantitative liver function reserve assessment in Asian countries. However, it is expensive and time consuming [4], also Child-Pugh score (CTP) which is the most widely used method for assessment of hepatic function, selecting patients for hepatectomy, and predicting postoperative mortality. However, some limitations of the CTP score in predicting the PHLF have been described [5].

Several studies concluded a significant association between chronic inflammatory diseases and increased incidence of malignancy including HCC [6]. The lymphocyte-monocyte ratio (LMR) is considered as a systemic inflammation-related marker and has recently been investigated in patients with solid tumors as a prognostic marker [7], including patients who had liver resection as a treatment for HCC [8].

It has been shown that the fibrosis score 4 (FIB-4) is effective in the assessment of liver fibrosis and cirrhosis [9, 10], in addition it is known as a good predictor of complications occurring after hepatectomy. However, the accuracy of FIB-4 to predict the short-term results of liver resection in patients with $\mathrm{HCC}$ had been investigated by few studies [11].

One of the non-invasive techniques for assessing liver stiffness is transient elastography (Fibroscan) [12]. By differentiating between the degree of liver fibrosis and cirrhosis that subsequently reflect the functional reserve of the liver which is important for patients with HCC undergoing liver resection [13].

However, until now no consensus was reached on the superiority of one of these methods to predict post-operative hepatic dysfunction after hepatic resection. To our knowledge, this is the first study on the predictive ability of LMR for PHLF.

This study aimed to determine the value of the LMR, FIB4 score and LSM using Fibroscan as pre-operative predictors of PHLF in Egyptian patients with post viral hepatitis C liver cirrhosis and HCC who underwent hepatectomy.

\section{Materials and Methods}

\subsection{Study Design and Patients Selection}

This was a prospective cohort study conducted in Egypt.
One hundred and seventeen (117) patients suffering from liver cirrhosis and HCC were screened for participation in this study during the period from September 2017 to September 2019. Of these, 50 patients with liver cirrhosis and $\mathrm{HCC}$ after $\mathrm{HCV}$ infection who underwent hepatectomy were enrolled. Patients were recruited from the multidisciplinary HCC clinic of Tropical Medicine and Infectious diseases Department in Tanta University Hospital and from the National Liver Institute, Menoufiya University, Egypt.

Before the beginning of this study an approval from faculty of medicine, Tanta University ethical committee was obtained (coded approval number: 31644/07/17) complying with the declaration of Helsinki (1975), in addition an informed written approval was signed by every patient before being enrolled in the study and a code number for each patient was used. All authors had full access to the study data; the final manuscript had been reviewed and accepted by all authors.

Eligibility criteria for treatment of HCC patients by liver resection included: Barcelona Clinic Liver Cancer (BCLC) stages 0 -A, adequate liver functional reserve, with Child Turcotte Pugh score (CTP) grade A, good performance status (score of $0-2$ ), infection with $\mathrm{HCV}$ and adequate residual liver volume determined by volumetric CT.

Patients received other treatments for HCC before hepatectomy, had vascular invasion, extrahepatic spread, other associated tumors, cardiac, pulmonary, renal or cerebral dysfunction were excluded from this study.

\subsection{Diagnosis and Definitions}

Liver cirrhosis was diagnosed on the basis of clinical, laboratory findings, imaging, Fibroscan and FIB-4 score. The CTP score was calculated based on its respective formula to assess liver disease severity [14]. HCC grading and resectability were determined by abdominal ultrasonography and contrastenhanced CT or MRI prior to liver resection. Diagnosis of HCC and cirrhosis were confirmed by post-operative pathological examination. HCC staging using BCLC staging system was done [15].

Clinically significant portal hypertension was established by: Detection of gastro-esophageal varices by upper endoscopy, splenic diameter more than $12 \mathrm{~cm}$ and/or count of platelet less than $100 \times 10^{9} / \mathrm{L}[16]$.

Definition of PHLF was made according to the " $50-50$ criteria" that is defined as prothrombin time (PT) $<50 \%$ (international normalized ratio (INR) $>1.7$ ) and serum bilirubin $>50 \mu \mathrm{mol} / \mathrm{L}$ $(>2.9 \mathrm{mg} / \mathrm{dL})$ on $5^{\text {th }}$ day post-operative [17].

While post-operatively mortality was defined as death within 30 days following surgery.

\subsection{Pre-Operative Assessment}

All of the patients in this study were subjected to detailed history, co-morbidities, clinical assessment, laboratory testing including (complete blood picture, liver profile, INR, renal function tests, serum alpha fetoprotein, $\mathrm{HBsAg}$, anti $\mathrm{HBc}$ 
total, anti-HCV antibody, quantitative PCR for HCV RNA), and radiological data (Ultrasonographic examination of abdomen and contrast-enhanced CT or MRI). While CT volumetry was done to ensure adequate residual liver volume which was 50 percent for patients with cirrhosis. All enrolled patients underwent upper gastrointestinal endoscopy and injection sclerotherapy or band ligation of esophageal varices was done if indicated.

\subsection{Calculation of Score Values}

Calculation of LMR was done according to equation (1):

Absolute lymphocyte count $\left(/ \mathrm{mm}^{3}\right.$ or $\left.\%\right) /$ absolute monocyte count $\left(/ \mathrm{mm}^{3}\right.$ or $\left.\%\right)(1)$ [18].

While the equation (2) used for FIB-4 score was:

Age $($ years $) \times\left(\right.$ AST $[\mathrm{U} / \mathrm{L}] /$ platelet count $\left[10^{9} / \mathrm{L}\right] \times \mathrm{ALT}$

$$
[\mathrm{U} / \mathrm{L}]^{1 / 2}(2)[19] \text {. }
$$

\subsection{Transient Elastography (Fibroscan)}

LSM was done by experienced operators using the Fibroscan (echosens-France) $502 \mathrm{M}$ probe within one week before hepatectomy. Only results with ten valid measurements, a success rate more than $60 \%$ and the interquartile range (IQR) less than $30 \%$ of the median value were considered valid. Kilopascals $(\mathrm{kPa})$ were the measuring units for these results [20].

\subsection{Surgical Technique}

Selected patients underwent hepatic resection of HCC focal lesions according to the standard protocols of surgery and anesthesia departments. Laparotomy was done by right subcostal or midline incision. Ultrasonography was used during operation to localize the tumor (s) and to confirm the relationships between the tumor (s) and their surrounding vessels. Either anatomical or non-anatomical resection, was selected on case-by-case basis. During the operation central venous pressure was frequently assessed by the anesthetist. Either Pringle maneuver or selective vascular occlusion of the hemiliver was chosen when needed. Bipolar coagulation, clips, and sutures were used for hemostasis.

\subsection{Post-Operative Assessment}

Regular follow up of all patients during hospitalization by physical examination and laboratory investigation to detect post-operative complications. Abdominal ultrasound, CT scan and chest X-ray were carried out when indicated. Assessment of PHLF was done on post-operative day 5 and post-operative mortality was reported.

\subsection{Statistical Analysis}

Statistical program for social science (SPSS) software version 22, 2015 USA was used to analyze data. Mean, standard deviation, median and range were used for description of quantitative variables. While frequency and percentages were used for qualitative variables. In order to compare a quantitative variable between two independent groups in parametric data Unpaired Student t test and MannWhitney $U$ test were used. Chi square test was used to compare a qualitative variable between two independent groups. $\mathrm{P}$ value was significant if $\leq 0.05$. For identification of independent predictors of PHLF multivariable logistic analysis was carried out using a logistic regression model. The area under the receiver operating characteristic (ROC) curve was calculated to measure the discriminatory power of the LMR, FIB-4 score, LSM with Fibroscan (by kpa value) as predictors of PHLF and its $95 \%$ confidence interval. To choose the best cut-off we selected the point with both maximum sensitivity and specificity on the ROC curve.

\section{Results}

One hundred and seventeen (117) patients diagnosed with liver cirrhosis and HCC were screened for enrollment in our study. Out of whom 67 patients were excluded thus, 50 patients with post HCV liver cirrhosis and HCC who underwent hepatectomy were enrolled. All patients in this study tested positive for $\mathrm{HCV}$ infection while negative for hepatitis B virus (HBV). PHLF developed in 14 patients $(28 \%)$ "Figure 1". So, patients were categorized into two groups:

Group I: included 36 patients who did not develop PHLF

Group II: included 14 patients who developed PHLF

The baseline characteristics of patient population according to development of PHLF are summarized in Table 1. In PHLF group, ten patients $(71.43 \%)$ had oesophageal varices grade 1 , nine patients $(64.29 \%)$ had performance status grade 1 , seven patients $(50 \%)$ had Child score A 6 , spleen size and tumor size were significantly increased when compared with patients without PHLF $(\mathrm{P} \leq 0.05)$ "Table 1".

Table 1. Baseline characteristics of patient population according to development of PHLF

\begin{tabular}{|c|c|c|c|c|}
\hline \multicolumn{2}{|l|}{ Parameters } & No PHLF $(n=36)$ & PHLF (n=14) & P-value \\
\hline \multicolumn{2}{|c|}{ Age (years); mean $\pm \mathrm{SD}$} & $57.06 \pm 7.92$ & $59 \pm 7.18$ & 0.428 \\
\hline \multicolumn{2}{|c|}{ Gender $[$ male, $\mathrm{n}(\%)]$} & $31(86.11 \%)$ & $11(78.57 \%)$ & 0.514 \\
\hline \multicolumn{2}{|c|}{ Hypertension } & $11(30.56)$ & $5(35.71)$ & 0.726 \\
\hline Oesophageal & No $n(\%)$ & $23(63.89 \%)$ & $4(28.57 \%)$ & \multirow{2}{*}{$0.024 *$} \\
\hline Varices & Yes (grade 1) n (\%) & $13(36.11 \%)$ & $10(71.43 \%)$ & \\
\hline \multicolumn{2}{|c|}{ Prothrombin concentration $(\%)$ mean \pm SD } & $85.89 \pm 12.18$ & $80.37 \pm 14.54$ & 0.281 \\
\hline \multicolumn{2}{|l|}{ INR mean \pm SD } & $1.13 \pm 0.15$ & $1.19 \pm 0.14$ & 0.252 \\
\hline
\end{tabular}




\begin{tabular}{|c|c|c|c|c|}
\hline \multicolumn{2}{|l|}{ Parameters } & No PHLF (n=36) & PHLF (n=14) & P-value \\
\hline \multicolumn{2}{|c|}{ Creatinine (mg/dl) mean \pm SD } & $0.86 \pm 0.18$ & $0.83 \pm 0.18$ & 0.691 \\
\hline \multicolumn{2}{|c|}{ AFP (ng/ml), median (IQR) } & $24.5(6.1-266.25)$ & $42(4.1-208.75)$ & 0.924 \\
\hline \multicolumn{2}{|c|}{ Hemoglobin $(\mathrm{g} / \mathrm{dl})$ mean \pm SD } & $13.47 \pm 1.5$ & $11.63 \pm 1.11$ & $<0.001 *$ \\
\hline \multicolumn{2}{|c|}{ Platelets $\left(\times 10^{3} / \mathrm{cmm}\right)$ mean \pm SD } & $162.31 \pm 61.7$ & $134.57 \pm 47.5$ & 0.137 \\
\hline \multicolumn{2}{|c|}{ Total bilirubin $(\mathrm{mg} / \mathrm{dl})$ mean $\pm \mathrm{SD}$} & $0.81 \pm 0.39$ & $1.1 \pm 0.21$ & $0.018 *$ \\
\hline \multicolumn{2}{|c|}{ Albumin $(\mathrm{g} / \mathrm{dl})$ mean $\pm \mathrm{SD}$} & $4.13 \pm 0.48$ & $3.49 \pm 0.29$ & $<0.001 *$ \\
\hline \multicolumn{2}{|c|}{ ALT (U/l) median (IQR) } & $39.5(22-65)$ & $50(28.5-77.75)$ & 0.161 \\
\hline \multicolumn{2}{|c|}{ AST (U/l) median (IQR) } & $41(27.75-54.25)$ & $73.4(42.5-87)$ & $0.008 *$ \\
\hline \multicolumn{2}{|c|}{ Spleen size $(\mathrm{cm})$ mean \pm SD } & $13.88 \pm 1.57$ & $15.3 \pm 0.89$ & $0.003 *$ \\
\hline \multicolumn{2}{|c|}{ Tumor size $(\mathrm{cm})$ mean \pm SD } & $4.97 \pm 2.63$ & $7.73 \pm 4.24$ & $0.008 *$ \\
\hline \multirow{2}{*}{$\begin{array}{l}\text { Performance } \\
\text { Status, n (\%) }\end{array}$} & PS0 & $25(69.44 \%)$ & $5(35.71 \%)$ & \multirow{2}{*}{$0.029 *$} \\
\hline & PS1 & $11(30.56 \%)$ & $9(64.29 \%)$ & \\
\hline \multirow{2}{*}{$\begin{array}{l}\text { BCLC stage, } \\
\text { n (\%) }\end{array}$} & BCLC 0 & $6(16.67 \%)$ & $0(0.00 \%)$ & \multirow{2}{*}{0.103} \\
\hline & BCLC A & $30(33.83 \%)$ & $14(100 \%)$ & \\
\hline \multirow{2}{*}{$\begin{array}{l}\text { Child score, } \\
\text { n (\%) }\end{array}$} & A5 & $30(83.33 \%)$ & $7(50 \%)$ & \\
\hline & A6 & $6(16.67 \%)$ & $7(50 \%)$ & $0.016 *$ \\
\hline \multicolumn{2}{|c|}{$\mathrm{LM}$ ratio mean $\pm \mathrm{SD}$} & $4.62 \pm 1.78$ & $2.52 \pm 0.52$ & $0.001 *$ \\
\hline \multicolumn{2}{|c|}{ FIB-4 score mean \pm SD } & $3.54 \pm 0.65$ & $5.18 \pm 1.99$ & $<0.001 *$ \\
\hline \multicolumn{2}{|c|}{ Fibroscan values $(\mathrm{kpa})$ mean $\pm \mathrm{SD}$} & $12.76 \pm 3.12$ & $24.34 \pm 11.08$ & $0.002 *$ \\
\hline
\end{tabular}

Abbreviations: AFP, alpha fetoprotein; ALT, Alanine transaminase; AST, Aspartate transaminase; BCLC, Barcelona clinic liver cancer; FIB-4 score, fibrosis score 4; kpa, kilopascal; INR, international normalizing ratio; IQR, Interquartile range; LM ratio, lymphocyte to monocyte ratio; PHLF, posthepatectomy liver failure; SD, standard deviation; $\mathrm{n}$, number *significant $(\mathrm{P} \leq 0.05)$.

As regards pre-operative laboratory investigations, hemoglobin concentration and serum albumin level showed significant decreases $(\mathrm{P}<0.001)$, while total bilirubin and AST were increased significantly in PHLF group when compared with patients without PHLF $(\mathrm{P} \leq 0.05)$ "Table 1".

The mean FIB-4 score was increased significantly in PHLF group ( $5.177 \pm 1.988$ vs. $3.542 \pm 0.654$ correspondingly) $(\mathrm{P}<0.001)$ and the mean LSM obtained by Fibroscan were significantly higher in PHLF group $(24.340 \pm 11.076$ vs. $12.761 \pm 3.122 \mathrm{kpa}$ respectively) $(\mathrm{P}=0.002)$ when compared with those without PHLF "Table 1".

In PHLF group, the mean LMR was significantly decreased compared with patients without PHLF $(2.517 \pm 0.515$ vs. $4.623 \pm 1.782$ respectively $) \quad(\mathrm{P}=0.001)$ "Table 1".

Regarding post-operative day 5 clinical features and laboratory investigations, patients who developed PHLF had hepatic encephalopathy, jaundice, lower limb edema and ascites which was significantly higher than those with no PHLF ( $\mathrm{P} \leq 0.05)$. In patients with PHLF, there were significant increases in INR, total bilirubin, ALT and AST levels $(\mathrm{P}<0.001)$ while serum albumin and platelets count significantly decreased in the in PHLF group $(\mathrm{P} \leq 0.05)$ when compared with patients without PHLF "Table 2".

Table 2. Post-operative day 5 assessment of the studied groups

\begin{tabular}{llll}
\hline Parameters & No PHLF $(\mathbf{n}=\mathbf{3 6})$ & PHLF $(\mathbf{n}=\mathbf{1 4})$ & P-value \\
\hline INR; mean \pm SD & $1.22 \pm 0.17$ & $1.88 \pm 0.06$ & $<0.001^{*}$ \\
Total bilirubin (mg/dl); mean \pm SD & $1.16 \pm 0.51$ & $3.44 \pm 0.42$ & $<0.001^{*}$ \\
Albumin (g/dl); mean \pm SD & $3.38 \pm 0.33$ & $2.74 \pm 0.29$ & $<0.001^{*}$ \\
ALT (U/l); mean \pm SD & $108.47 \pm 41.95$ & $209.79 \pm 96.48$ & $<0.001^{*}$ \\
AST (U/l); mean \pm SD & $87.56 \pm 35.7$ & $178.57 \pm 72.3$ & $<0.001^{*}$ \\
Platelets (x10/c.mm); mean \pm SD & $139.36 \pm 51.75$ & $98.14 \pm 37.48$ & $0.017^{*}$ \\
Hepatic encephalopathy, $(\%)$ & $0(0 \%)$ & $2(14.29 \%)$ & $0.021^{*}$ \\
Lower limb edema, $\mathrm{n}(\%)$ & $3(8.33 \%)$ & $14(100 \%)$ & $<0.001^{*}$ \\
Ascites, $\mathrm{n}(\%)$ & $1(2.78 \%)$ & $13(92.86 \%)$ & $<0.001^{*}$ \\
\hline
\end{tabular}

Abbreviations: ALT, Alanine transaminase; AST, Aspartate transaminase; INR, international normalizing ratio; PHLF, posthepatectomy liver failure; SD, standard deviation; $\mathrm{n}$, number; *significant $(\mathrm{P} \leq 0.05)$.

Post-operative complications occurred in 24 patients (48\%) and were more common in patients who developed PHLF when compared with those without PHLF (14 vs. 10 patients) $(\mathrm{P}=0.001)$. The most common complication after PHLF was pleural effusion, which occurred in 7 patients (14\%) of PHLF group. Two patients died during 30 days of operation as a result of PHLF, with a mortality rate of $4 \%$ "Figure $1 "$. 


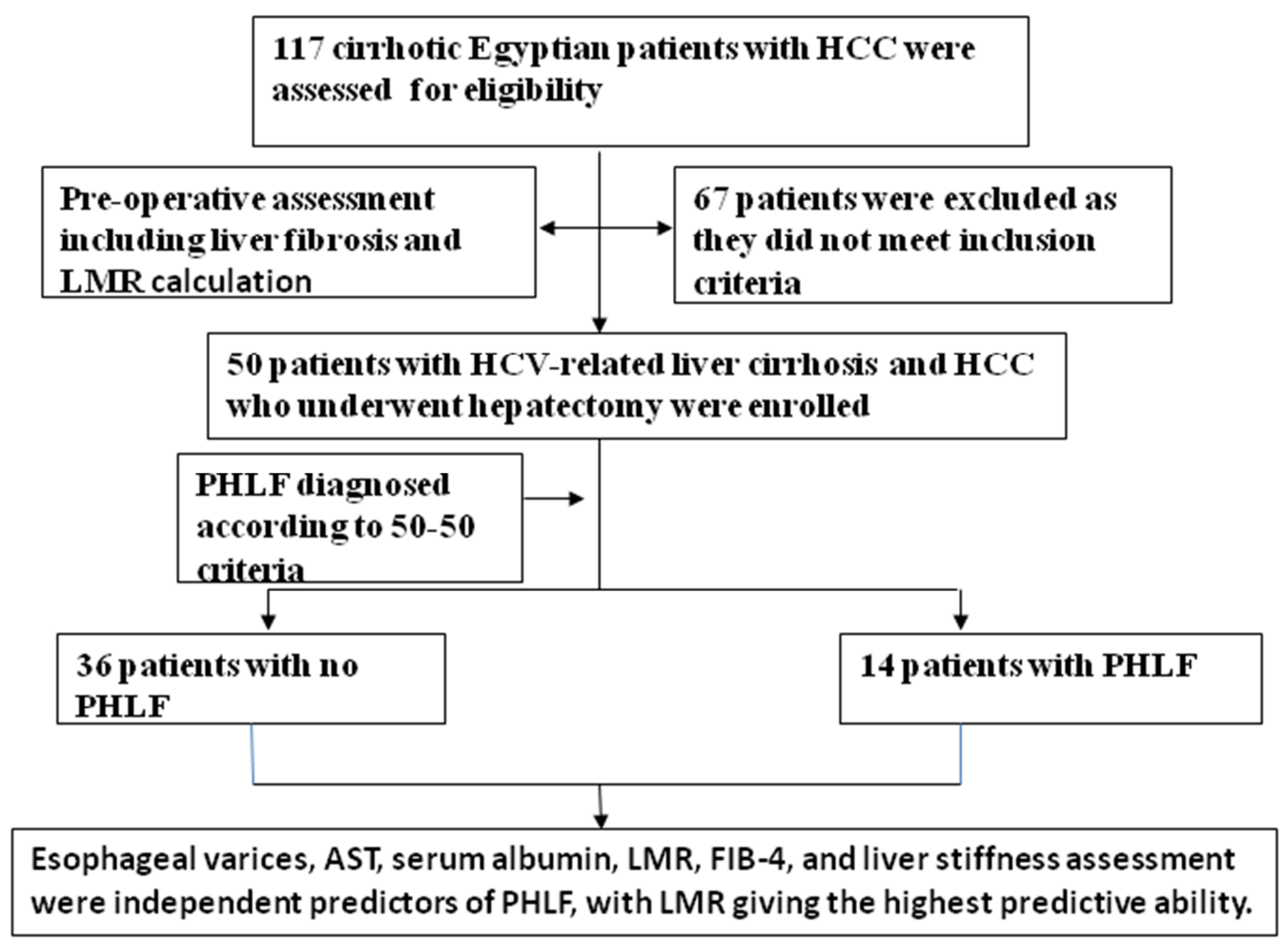

Figure 1. Consort flow diagram showing patients enrollment and follow up

Abbreviations: HCC, hepatocellular carcinoma; LMR, lymphocyte/ monocyte ratio; PHLF, post-hepatectomy liver failure; AST, aspartate aminotransferase; FIB-4, fibrosis score 4.

A multivariate logistic regression analysis showed that, presence of oesophageal varices, serum AST, serum albumin, LMR, FIB-4 score, and LSM with Fibroscan $(\mathrm{P}=0.040 ; 0.038 ; 0.024 ; 0.001 ; 0.022,0.016$ respectively) were independent predictors for PHLF "Table 3".

Table 3. Univariate and multivariate analyses for the preoperative predictors of post-hepatectomy liver failure

\begin{tabular}{lllll}
\hline \multirow{2}{*}{ Variables } & Univariate analysis & \multicolumn{3}{l}{ Multivariate analysis } \\
\cline { 2 - 5 } & OR (95\% C. I) & P-value & OR (95\% C. I) & P-value \\
\hline Oesophageal varices & $4.423(1.153-5.955)$ & $0.030^{*}$ & $2.421(1.50-6.964)$ & $0.040^{*}$ \\
Spleen size (cm) & $2.311(1.249-4.275)$ & $0.008^{*}$ & $1.768(0.883-3.539)$ & 0.108 \\
Hemoglobin & $0.380(0.209-0.690)$ & $0.001^{*}$ & $0.364(0.128-1.032)$ & 0.057 \\
Total bilirubin & $7.523(1.270-14.579)$ & $0.026^{*}$ & $0.685(0.041-1.322)$ & 0.791 \\
Albumin & $0.022(0.002-0.245)$ & $0.002^{*}$ & $0.005(0.000-0.499)$ & $0.024^{*}$ \\
AST & $1.023(1.004-1.042)$ & $0.018^{*}$ & $1.073(1.004-1.147)$ & $0.038^{*}$ \\
Tumor size (cm) & $1.273(1.044-1.552)$ & $0.017^{*}$ & $1.218(0.935-1.585)$ & 0.143 \\
Performance status & $4.091(1.111-15.057)$ & 0.074 & - & - \\
Child score & $5.012(1.276-9.597)$ & $0.032^{*}$ & $2.748(1.566-5.596)$ & 0.066 \\
LMR & $2.363(1.353-4.125)$ & $<0.031^{*}$ & $1.68(3.501-8.071)$ & $0.001^{*}$ \\
FIB-4 score & $5.3301 .848-10.369)$ & $0.002^{*}$ & $7.892(1.323-9.422)$ & $0.022^{*}$ \\
Fibroscan values (kpa) & $6.50(3.377-12.512)$ & $0.006^{*}$ & $5.98(2.202-6.480)$ & $0.016^{*}$ \\
\hline
\end{tabular}

Abbreviations: AST, Aspartate transaminase; C. I, confidence interval; FIB-4 score, fibrosis score 4; kpa, kilopascal; LM ratio, lymphocyte to monocyte ratio; OR, odds ratio; *significant $(\mathrm{P} \leq 0.05)$.

According to the ROC curve analysis, LMR yielded the best accuracy for predicting PHLF at cutoff $<3.33$ [AUC $=0.940$; sensitivity $=93.65 \%$; specificity $=94.44 \%$; positive predictive value $=86.67 \%$; negative predictive value $=97.14 \%]$. FIB-4 score and LSM obtained by Fibroscan had lower AUC (0.886 and 0.875$)$ respectively "Table 4, Figure $2 "$. 
Mona Nasef et al.: Fibroscan and FIB-4 Assessment Versus Lymphocyte / Monocyte Ratio as Predictors of Post-Hepatectomy Liver Failure in HCV Egyptian Patients with Hepatocellular Carcinoma

30

25

20

15

5

0

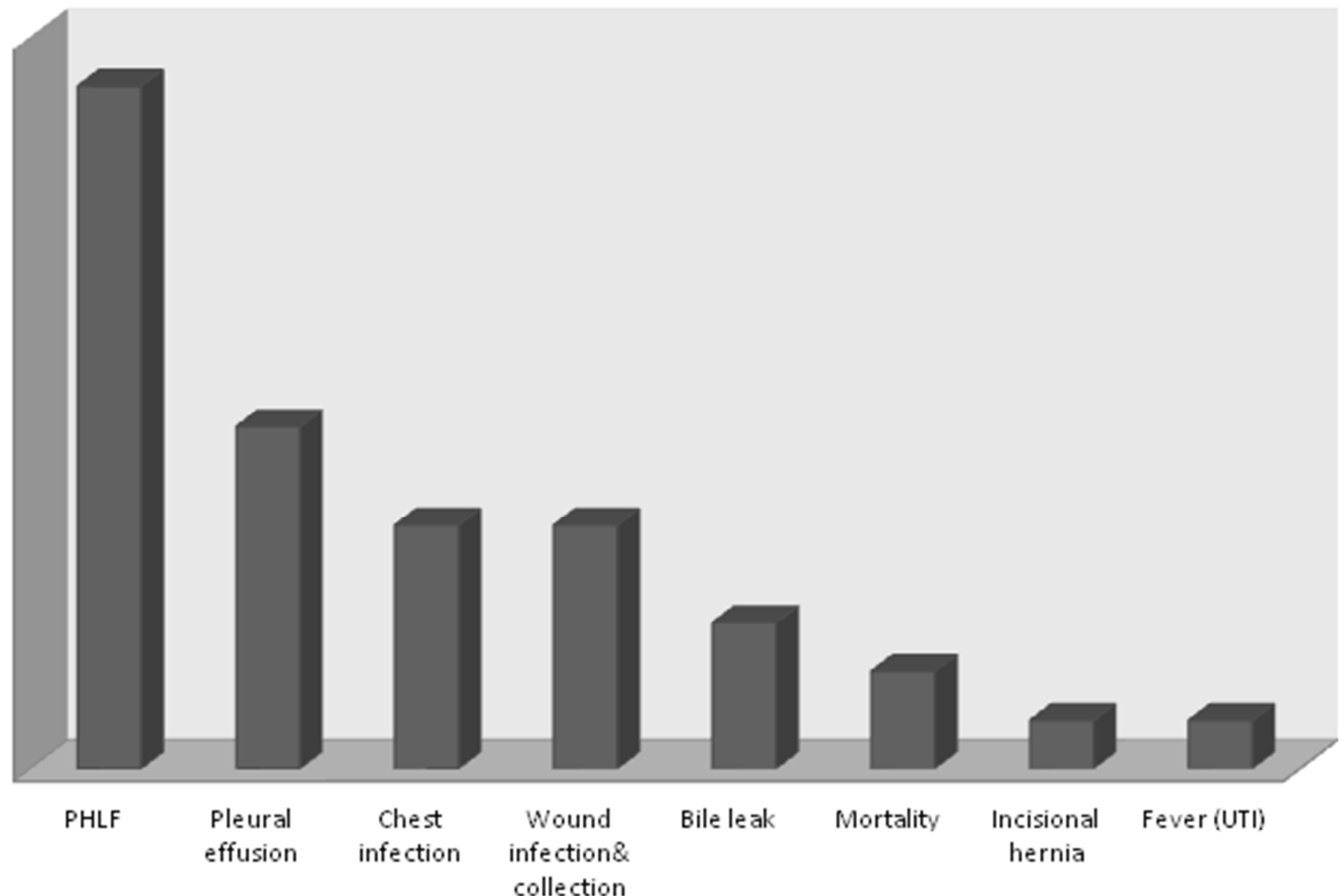

Abbreviations: PHLF, post-hepatectomy liver failure; UTI, urinary tract infection

Figure 2. Post-operative complications and mortality in the studied patients.
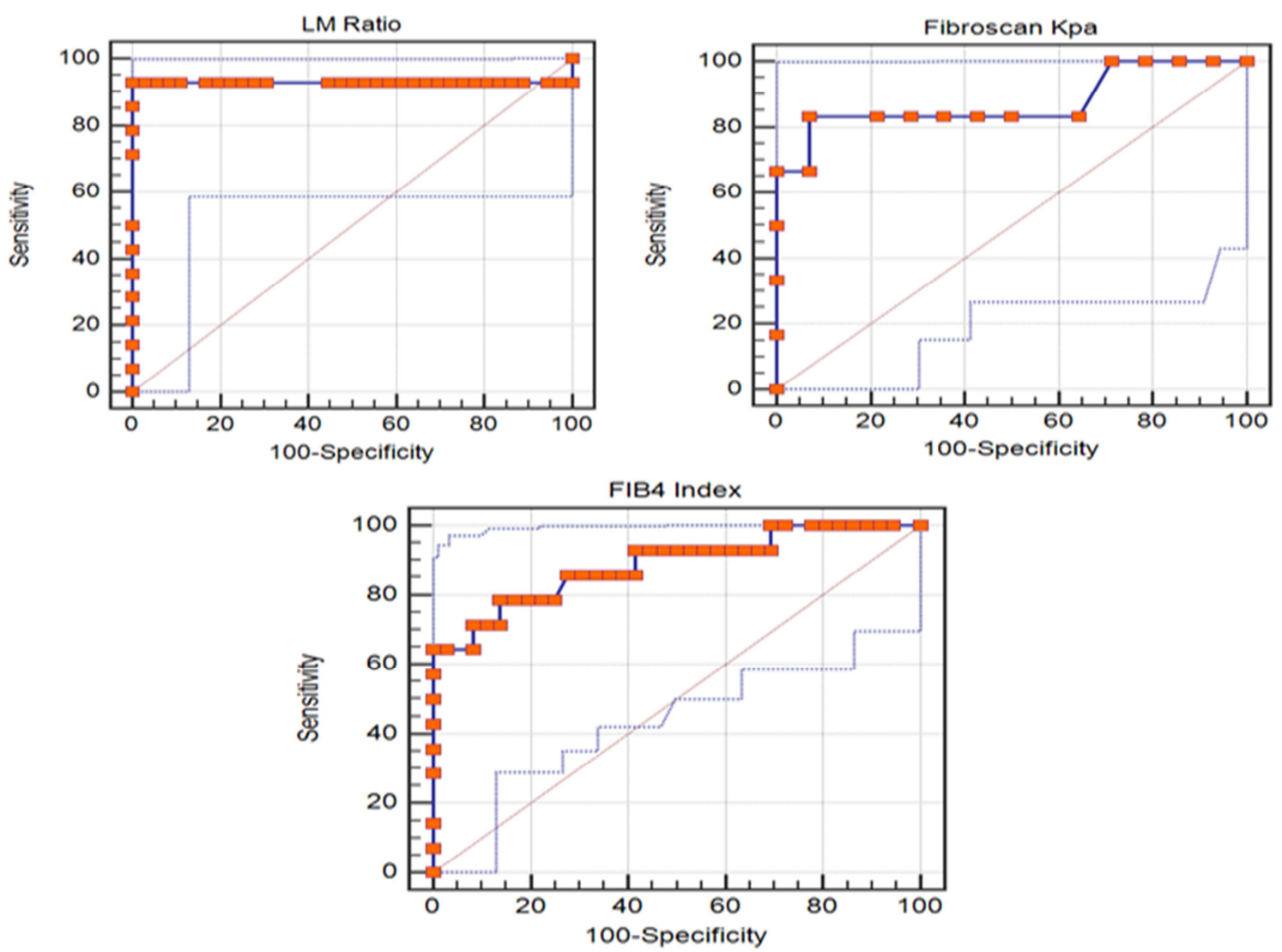

Abbreviations: LMR, lymphocyte to monocyte ratio; FIB-4 score, fibrosis score 4; kpa, kilopascal.

Figure 3. ROC curves for LMR, FIB-4 and Fibroscan as predictors of post-hepatectomy liver failure. LMR had the best accuracy for predicting PHLF. 
Table 4. Receiver operating characteristic (ROC) curves for LMR, FIB-4 index, Fibroscan as predictors of post-hepatectomy liver failure.

\begin{tabular}{lllllll}
\hline & Cutoff & Sensitivity\% & Specificity\% & PPV\% & NPV\% & AUC \\
\hline LMR & $<3.33$ & 93.65 & 94.44 & 86.67 & 97.14 & 0.940 \\
FIB-4 score & $>3.94$ & 71.43 & 91.67 & 76.9 & 89.2 & 0.886 \\
Fibroscan values (kpa) & $>15.5$ & 83.33 & 92.86 & 83.3 & 92.9 & 0.875 \\
\hline
\end{tabular}

Abbreviations: AUC, area under the curve; FIB-4 score, fibrosis score 4; kpa, kilopascal; LM ratio, lymphocyte to monocyte ratio; NPV, negative predictive value; PPV, positive predictive value.

\section{Discussion}

This study was a prospective cohort which was designed to determine the value of the LMR, FIB-4 score and LSM estimated by Fibroscan as pre-operative predictors of PHLF in Egyptian patients suffering from liver cirrhosis and HCC post $\mathrm{HCV}$ infection who underwent hepatectomy.

To the best of our information, this is the first study that assessed the pre-operative LMR as a predictor for PHLF. Only patients suffering from $\mathrm{HCC}$ related to $\mathrm{HCV}$ infection were enrolled in this study in order to evade possible confounding factors due to other etiologies.

In this study, a multivariate analysis revealed that preoperative LMR, FIB-4 score and LSM using Fibroscan were independent predictors for PHLF.

The optimal pre-operative LMR cut-off value established for predicting PHLF by ROC curve analysis was $<3.33$ $($ AUC $=0.940)$. The mechanisms that LMR can predict PHLF could be related to a complex inflammatory response. LMR is a systemic inflammatory marker which includes lymphocytes and monocytes. Lymphocytes can protect against tumorigenesis and recurrence by inhibiting tumor cells proliferation through cytokines generation and causing cytotoxic death in cells [21]. The reduced number of lymphocyte may indicate a weak antitumor reaction leading to poor clinical outcome [22]. Another important element of immunity in human body is Monocytes, as they promote tumorigenesis by suppressing local immune systems [23]. Additionally they can differentiate into tumor-associated macrophages that promote the development of tumors, angiogenesis, invasion, and metastasis [24].

Lymphocytes and monocytes have a crucial role in the biological behavior of liver cancer, for example; the onset, differentiation, and metastases [25-27]. Several studies have showed the association between pre-operative low LMR and size, vascular invasion and staging of tumors. High LMR reflects intensive immune system anti-tumor effect, represents lymphocytosis and/or monocytopenia. On the other hand low LMR stand for the immune system depressed state. Consequently, HCC patients with high LMR have postoperative favorable outcomes, and vice versa [28].

Pre-operative LMR accurately predicts PHLF and is potentially a good indicator for selecting suitable HCC patients who will undergo hepatectomy.

As liver fibrosis and cirrhosis is detected in most of HCC patients and has a major role in the PHLF occurrence [29]. Therefore, the precise assessment of liver fibrosis and cirrhosis prior to surgery is essential. FIB-4 score and LSM estimated by Fibroscan are noninvasive methods used for the estimation of liver stiffness [12].

Our pre-operative FIB-4 score cut-off value of $>3.94$ (AUC $=0.886$ with $71.43 \%$ sensitivity and $91.67 \%$ specificity) accurately predicted PHLF in our study. This was close to the data found by Zhou et al who reported the optimal cutoff value of the FIB-4 score for predicting PHLF was 4.16 with $65.2 \%$ sensitivity and $83.3 \%$ specificity. Increased FIB-4 score value indicates advanced liver fibrosis or cirrhosis which coincided with poor liver function and poor postoperative outcome such as failure of the liver, worsening encephalopathy or ascites [30].

According to ROC curve analysis, the best cutoff value to predict PHLF for the pre-operative LSM using Fibroscan was $>15.5$ (kpa) $(A U C=0.875$ with $83.33 \%$ sensitivity and $92.86 \%$ specificity). This was in agreement with the findings of another Egyptian study that identified LSM value $\geq 15.4$ $(\mathrm{kPa})$ to have the optimal cutoff value for predicting PHLF with $100 \%$ sensitivity and $100 \%$ specificity [31]. Lei et al reported that, LSM cutoff value of $14(\mathrm{kPa})$ with $94.6 \%$ sensitivity and $67.6 \%$ specificity as the optimal cutoff for development of PHLF in Chinese patients [32].

There is a little information regarding hepatic fibrosis effect on PHLF. In the fibrotic liver, it is proposed that regeneration is a process mediated by progenitor cells, in contrast to replication of mature liver cells in the noncompromised liver. Following partial hepatectomy, liver cell regeneration impairment and subsequent hepatic dysfunction are caused by inefficient induction of mediators of cell cycle transition, necrosis of liver cell, and a marked fibrogenic response [33, 34]. Reduced activity of innate and adaptive immune system and enhanced bacterial translocation increase the vulnerability of the fibrotic liver [35].

Pre-operative FIB-4 score and LSM using Fibroscan are useful to predict PHLF in patients with HCC and should be routinely assessed in those patients who will undergo hepatectomy.

The present study has some limitations. All enrolled HCC patients had HCV liver cirrhosis in addition small sample size. So, the results of this study can't be applied to patients without chronic liver disease. Therefore, further validation is required from a large population with different etiologies.

In conclusion, the pre-operative LMR has a superior predictive ability for PHLF in patients with HCV-related HCC undergoing hepatectomy compared with FIB-4 score and LSM using Fibroscan and should be included routinely in the pre-operative workup of HCC patients being candidate 
for hepatectomy.

\section{Conflicts of Interest}

The authors declare that they have no competing interests.

\section{Funding}

None.

\section{Acknowledgements}

Declared none.

\section{References}

[1] Holah, N. S, El-Azab, D. S., Aiad, H. A., Sweed, D. M (2015) Hepatocellular carcinoma in Egypt: epidemiological and histopathological properties, Menoufia Medical Journal, 28: 718-724.

[2] McCormack, L., Petrowsky, H., Jochum, W., Furrer, K., Clavien, P. A (2007) Hepatic steatosis is a risk factor for postoperative complications after major hepatectomy: a matched case-control study, Annals of surgery, 245: 923-930.

[3] Hong, G., Suh, K. S., Suh, S. W., Yoo, T., Kim, H., et al. (2016) Alpha-fetoprotein and 18F-FDG positron emission tomography predict tumor recurrence better than Milan criteria in living donor liver transplantation. Journal of hepatology, 64: 852-859.

[4] Vitale, A., Burra, P., Frigo, A. C, Trevisani, F., Farinati, F., et al. (2015) Survival benefit of liver resection for patients with hepatocellular carcinoma across different Barcelona Clinic Liver Cancer stages: a multicentre study. Journal of hepatology, 62: 617-624.

[5] Wang, Y. Y, Zhong, J. H., Su, Z. Y, Huang, J. E, Lu, S. D, et al. (2016) Albumin-bilirubin versus Child- Pugh score as a predictor of outcome after liver resection for hepatocellular carcinoma. British Journal of Surgery, 103: 725-734.

[6] Berasain, C., Castillo, J., Perugorria, M. J., Latasa, M. U., Prieto, J., (2009) Inflammation and liver cancer: new molecular links. Ann N Y Acad Sci, 1155: 206-221.

[7] Ozawa, T., Ishihara, S., Kawai, K., Kazama, S., Yamaguchi, H., et al. (2015) Impact of a lymphocyte to monocyte ratio in stage IV colorectal cancer. J Surg Res, 199: 386-392.

[8] Wu, S. J, Lin, Y. X, Ye, H., Li, F. Y, Xiong, X. Z, et al. (2016) Lymphocyte to monocyte ratio and prognostic nutritional index predict survival outcomes of hepatitis B virus-associated hepatocellular carcinoma patients after curative hepatectomy. Journal of surgical oncology, 114: 202-210.

[9] Xiao, G., Yang, J. and Yan, L. (2015) Comparison of diagnostic accuracy of aspartate aminotransferase to platelet ratio index and fibrosis-4 index for detecting liver fibrosis in adult patients with chronic hepatitis $\mathrm{B}$ virus infection: a systemic review and meta-analysis. Hepatology, 61: 292-302.
[10] Zhang, E., Zhang, Z. Y., Wang, S. P., Xiao, Z. Y., Gu, J., et al. (2016) Predicting the severity of liver cirrhosis through clinical parameters. J Surg Res, 204: 274-281

[11] Dong, J., Xu, X. H, Ke, M. M, Xiang, J. X, Liu, W. Y, et al. (2016) The FIB-4 score predicts post-operative short-term outcomes of hepatocellular carcinoma fulfilling the milan criteria. European Journal of Surgical Oncology (EJSO), 42: 722-727.

[12] Sandrin, L., Fourquet, B., Hasquenoph, J. M., Yon, S., Fournier, C., et al. (2003) Transient elastography: a new noninvasive method for assessment of hepatic fibrosis. Ultrasound Med Biol, 29: 1705-1713.

[13] Cescon, M., Colecchia, A., Cucchetti, A., Peri, E., Montrone, L., et al. (2012) Value of transient elastography measured with fibroscan in predicting the outcome of hepatic resection for hepatocellular carcinoma. Ann. Surg, 256: 706-712.

[14] Vauthey, J. N, Dixon, E., Abdalla, E. K., Helton, W. S., Pawlik, T. M, et al. (2010) Pretreatment assessment of hepatocellular carcinoma: expert consensus statement. Hepato-PancreatoBiliary Association, 12: 289-299.

[15] Forner, A., Llovet, J. M, and Bruix, J. (2012) Hepatocellular carcinoma. Lancet, 379: 1245-1255.

[16] Berzigotti, A., Reig, M., Abraldes, J. G, Bosch, J., and Bruix, J. (2015) Portal hypertension and the outcome of surgery for hepatocellular carcinoma in compensated cirrhosis: a systematic review and meta-analysis. Hepatology, 61: 526-536.

[17] Balzan, S., Belghiti, J., Farges, O., Ogata, S., Sauvanet, A., Delefosse, D., et al. (2005) The "50-50 criteria" on postoperative day 5: an accurate predictor of liver failure and death after hepatectomy. Ann Surg, 242: 824-828, discussion 828-829.

[18] Neofytou, K., Smyth, E. C., Giakoustidis, A., Khan, A. Z, Williams, R., et al. (2015) The pre-operative lymphocyte-tomonocyte ratio is prognostic of clinical outcomes for patients with liver-only colorectal metastases in the neoadjuvant setting. Annals of surgical oncology, 22: 4353-4362.

[19] Suh, B., Park, S., Shin, D. W., Yun, J. M, Yang, H. K, et al. (2015) High liver fibrosis index FIB-4 is highly predictive of hepatocellular carcinoma in chronic hepatitis B carriers. Hepatology, 61: 1261-1268.

[20] S irli, R., Sporea, I., Bota, S., et al. (2013) Factors influencing reliability of liver stiffness measurements using transient elastography (M-probe)- monocentric experience. Eur J Radiol, 82 (8): e313-6.

[21] Dun, G. P, Old, L. J, and Schreiber, R. D. (2004) The immunobiology of cancer immunosurveillance and immunoediting. Immunity, 21: 137-148.

[22] Santoiemma, P. P, and Powell, DJ. Jr. (2015) Tumor infiltrating lymphocytes in ovarian cancer. Cancer Biol Ther, 16: $807-820$

[23] Gabrilovich, D. I., and Nagaraj, S. (2009) Myeloid-derived suppressor cells as regulators of the immune system. Nat Rev Immunol, 9: 162-174.

[24] Condeelis, J., and Pollard, J. W. (2006) Macrophages: obligate partners for tumor cell migration, invasion, and metastasis. Cell, 124: 263-266. 
[25] Unitt, E., Marshall, A., Gelson, W., Rushbrook, S. M, Davies, S., et al. (2006) Tumour lymphocytic infiltrate and recurrence of hepatocellular carcinoma following liver transplantation. J Hepatol, 45: 246-253.

[26] Gao, Q., Qiu, S. J., Fan, J., Zhou, J., Wang, X. Y., et al. (2007) Intratumoral balance of regulatory and cytotoxic $T$ cells is associated with prognosis of hepatocellular carcinoma after resection. J Clin Oncol, 25: 2586-2593.

[27] Mano, Y., Aishima, S., Fujita, N., Tanaka, Y., Kubo, Y., et al. (2013) Tumor-associated macrophage promotes tumor progression via STAT3 signaling in hepatocellular carcinoma. Pathobiology, 80: 146-154.

[28] Shi, S., Chen, Q., Ye, L., Yin, D., Li, X., et al. (2017) Prognostic value of systemic inflammation score in patients with hepatocellular carcinoma after hepatectomy. Oncotarget, 8: 79366-79375.

[29] Thompson Coon, J., Rogers, G., Hewson, P., Wright, D., Anderson, R., et al. (2007) Surveillance of cirrhosis for hepatocellular carcinoma: systematic review and economic analysis. Health Technol Assess, 11: 1-206.

[30] Zhou, P., Chen, B., Miao, X. Y, Zhou, J. J, Xiong, L., et al. (2019) Comparison of FIB-4 score and Child-Pugh Score in Predicting the Outcome of Hepatic Resection for Hepatocellular Carcinoma. Journal of Gastrointestinal Surgery, 23: $1-9$.
[31] Hegazy, O., Allam, M., Sabry, A., Kohla, M. A. S., Abogharbia, W., et al. (2019) Liver stiffness measurement by transient elastography can predict outcome after hepatic resection for hepatitis $\mathrm{C}$ virus-induced hepatocellular carcinoma. The Egyptian Journal of Surgery, 38: 313-318.

[32] Lei, J. W, Ji, X. Y, Hong, J. F, Li, W. B, Chen, Y., et al. (2017) Prediction of posthepatectomy liver failure using transient elastography in patients with hepatitis B related hepatocellular carcinoma. BMC gastroenterology, 17: 171.

[33] Kuramitsu, K., Sverdlov, D. Y, Liu, S. B, Csizmadia, E., Burkly, L., et al. (2013) Failure of fibrotic liver regeneration in mice is linked to a severe fibrogenic response driven by hepatic progenitor cell activation. The American journal of pathology, 183: 182-194.

[34] Kato, A., Bamba, H., Shinohara, M., Yamauchi, A., Ota, S., et al. (2005) Relationship between expression of cyclin D1 and impaired liver regeneration observed in fibrotic or cirrhotic rats. Journal of gastroenterology and hepatology, 20: 11981205.

[35] Jalan, R., Fernandez, J., Wiest, R., Schnabl, B., Moreau, R., et al. (2014) Bacterial infections in cirrhosis: a position statement based on the EASL Special Conference 2013. Journal of hepatology, 60: 1310-1324. 